\title{
硫掺杂碳纳米笼的制备及其氧还原性能研究
}

\author{
王立伟 ${ }^{a}$ 冯瑞 ${ }^{a}$ 夏婧竹 ${ }^{a}$ 陈盛 ${ }^{a}$ 吴强*,,$b$ \\ 杨立军 $a$ 王喜章*, $a, b$ 胡征 $a, b$ \\ ( ${ }^{a}$ 介观化学教育部重点实验室 南京大学化学化工学院 南京 210093) \\ ( ${ }^{b}$ 南京大学(苏州)高新技术研究院 苏州 215123)
}

\begin{abstract}
摘要 以噻吩和苯为硫源和碳源、以 $\mathrm{MgO}$ 为模板, 通过化学气相沉积法制得了硫掺杂碳纳米笼, 具有比表面积大、孔 道结构丰富和石墨化程度较好等特点. 通过改变噻吩的用量可在 $0 \sim 3.45$ at\%范围内调变其硫掺杂量. 系统考察了硫的 掺杂量对于碳纳米笼氧还原性能的影响, 结果表明: 硫含量为 $0.84 \mathrm{at} \%$ 的 SCNCs 具有最佳的氧还原性能, 随着硫掺杂 量增多其氧还原性能逐渐变差, 当硫的掺杂量高于 $1.61 \mathrm{at} \%$ 时甚至不如未掺杂的碳纳米笼. 这对于通过调变掺杂元素 种类、含量及掺杂构型优化碳基无金属氧还原催化剂具有参考价值.
\end{abstract}

关键词 碳纳米笼; 硫掺杂; 氧还原; 无金属; 燃料电池

\section{Synthesis and Electrocatalytic Oxygen Reduction Performance of the Sulfur-Doped Carbon Nanocages}

\author{
Wang, Liwei ${ }^{a} \quad$ Feng, Rui $^{a} \quad$ Xia, Jingzhu $^{a} \quad$ Chen, Sheng ${ }^{a}$ \\ $\mathrm{Wu}$, Qiang, ${ }^{*, a, b}$ Yang, Lijun $^{a} \quad$ Wang, Xizhang*,a,b $\quad$ Hu, Zheng ${ }^{a, b}$ \\ $\left({ }^{a}\right.$ Key Laboratory of Mesoscopic Chemistry of MOE, School of Chemistry and Chemical Engineering, \\ Nanjing University, Nanjing 210093) \\ $\left({ }^{b}\right.$ High-Tech Research Institute of Nanjing University (Suzhou), Suzhou, Jiangsu, 215123)
}

\begin{abstract}
The sluggish oxygen reduction reaction (ORR) is the bottleneck in the development of fuel cells, and replacing precious and nondurable Pt catalysts by the material with low cost, high activity and good stability is a main challenge. Carbon-based metal-free ORR electrocatalysts have become a promising alternative of commercial $\mathrm{Pt} / \mathrm{C}$ catalyst due to their superior catalytic activity, high stability and low cost. Recent studies revealed that the doping of N, B, P or S atoms could boost the ORR electrocatalytic performance of carbon nanomaterials, and the catalytic activities were highly dependent on the doping elements, amounts and microstructures. In this study, sulfur-doped carbon nanocages (SCNCs) were synthesized by chemical vapor deposition method using in situ generated $\mathrm{MgO}$ as template and thiophene/benzene as precursors. The resultant SCNCs possessed high specific surface area of $c a .1000 \mathrm{~m}^{2} \cdot \mathrm{g}^{-1}$, abundant pore structure and superior graphitization degree. The X-ray photoelectron spectroscopy result showed sulfur atoms were doped into the carbon framework as the $\mathrm{C}-$ $\mathrm{S}-\mathrm{C}$ moieties. The content of sulfur in the SCNCs was adjusted in the range of $0 \sim 3.45$ at $\%$ by changing the amount of thiophene in the precursor. All the SCNCs samples had comparable specific surface area and similar pore structure. As an electrocatalyst for oxygen reduction reaction (ORR) in alkaline medium, the SCNCs exhibited a sulfur-content-dependent performance. The SCNCs with sulfur content of 0.84 at $\%$ demonstrated the optimal ORR performance. With further increasing the sulfur content, the ORR performance of the SCNCs gradually degraded and even inferior to that of the pure CNCs when the sulfur content was higher than $1.61 \mathrm{at} \%$. In addition, the SCNCs showed better stability and immunity to methanol crossover than the $\mathrm{Pt} / \mathrm{C}$ catalyst. This result is suggestive for designing advanced metal-free ORR electrocatalysts by regulating the species and content of dopants and doping microstructures.

Keywords carbon nanocages; sulfur doping; oxygen reduction; metal-free; fuel cells
\end{abstract}

\section{1 引言}

燃料电池是将化学能转化为电能的装置, 不受卡诺
循环的限制，能源转化率高且环境友好，是未来新能源 体系的重要组成部分 ${ }^{[1,2]}$. Pt/C 是目前使用最广泛的商用 燃料电池催化剂, 但 Pt 的储量有限、价格昂贵, 对一氧

*E-mail:wqchem@nju.edu.cn; wangxzh@nju.edu.cn; Tel.: 025-83593696; Fax: 025-83593696

Received May 27, 2014; published September 15, 2014.

Project supported by the National Natural Science Foundation of China (Nos. 51232003, 21473089, 21173115, 21173114, 21203092), “973” program (No. 2013CB932902) and Jiangsu Province Science and Technology Support Project (No. BE2012159), Suzhou Science and Technology Plan Projects (No. ZXG2013025).

项目受国家自然科学基金(Nos. 51232003，21473089，21173115，21173114，21203092)、“973”项目(No. 2013CB932902)、江苏省科技支撑项目(No. BE2012159)和苏州市科技计划项目(No. ZXG2013025)资助. 
化碳和燃料(甲醇)敏感，这限制了燃料电池的大规模商 业化应用 ${ }^{[3,4]}$. 燃料电池面临的主要挑战是设计和制备 廉价、稳定的氧还原催化剂 ${ }^{[5]}$. 目前, 人们致力于开发低 铂用量甚至无铂的氧还原催化剂 ${ }^{[6]}$. 碳基无金属氧还原 催化剂因其成本低、活性高、稳定性好等优点受到广泛 关注 ${ }^{[7]}$. 研究表明: 多电子、电负性高的 $\mathrm{N}$ 原子掺杂到 $\mathrm{sp}^{2}$ 碳材料中可以显著提高氧还原活性, 已在氮掺杂碳 纳米管 ${ }^{[8]}$ 、氮掺杂石墨烯 ${ }^{[9]}$ 、氮掺杂模板碳 ${ }^{[10]}$ 和 $\mathrm{g}-\mathrm{C}_{3} \mathrm{~N}_{4}{ }^{[11]}$ 等材料中得以体现. 我们课题组发现少电子、电负性低 的 $\mathrm{B}$ 原子掺杂同样使碳纳米管具有较好的氧还原催化 性能 ${ }^{[12]}$. 近年来, 通过调控 $\mathrm{sp}^{2}$ 碳纳米材料的掺杂开发 无金属氧还原电催化剂成为研究热点, 例如, 文献报道 $\mathrm{S}^{[13 \sim 15]}, \mathrm{P}^{[16]}, \mathrm{I}^{[17]}, \mathrm{Si}^{[18]}$ 等原子掺杂都能在一定程度上提 高碳材料的氧还原性能. 我们课题组在探究 $\mathrm{B}, \mathrm{N}$ 共掺 杂碳纳米管的氧还原性能时发现, 掺杂微结构对氧还原 性能有决定性的作用 ${ }^{[19]}$ : B, N 相互分离的纳米管具有优 异的氧还原活性, 而 $\mathrm{B}, \mathrm{N}$ 相邻的纳米管失去了氧还原 活性. 可见, 掺杂对氧还原性能的调变取决于掺杂元素 和掺杂构型等多种因素.

最近, 我们课题组发展了以 $\mathrm{MgO}$ 为模板、以苯或吡 啶为前驱物, 通过化学气相沉积过程制备碳纳米笼 $(\mathrm{CNCs})^{[20]}$ 及氮掺杂碳纳米笼( NCNCs) 的方法 ${ }^{[21]}$. 这类 纳米笼具有大比表面积、丰富孔道结构、高石墨化程度 等特点, 氮掺杂碳纳米笼展现出优异的氧还原电催化性 能. 本文以噻吩和苯为前驱体, 通过相似路线合成了比 表面积大、孔道结构丰富、石墨化程度较好的硫掺杂碳 基纳米笼(SCNCs), 在 $0 \sim 3.45$ at $\%$ 范围调变了硫的含量, 系统考察了硫含量对氧还原性能的影响, 发现少量 $\mathrm{S}$ 掺 杂有利于改善氧还原性能, 当 $\mathrm{S}$ 含量高于 $1.61 \mathrm{at} \%$ 时反 而降低了碳材料的氧还原性能. 这个结果对通过调变掺 杂元素种类、含量及掺杂构型优化碳基无金属氧还原催 化剂具有参考价值.

\section{2 结果与讨论}

通过改变前驱物中噻吩和苯的体积比, 我们制备了 系列 SCNCs 样品, 命名为 $\mathrm{S}_{n} \mathrm{CNC}$, 其中 $n$ 对应于噻吩的 体积百分比. 以未掺杂碳纳米笼 $\mathrm{S}_{0} \mathrm{CNC}$ 和硫掺杂量最 大样品 $\mathrm{S}_{100} \mathrm{CNC}$ 为代表, 其结构和成分表征结果见图 1. 图 1a, 1b 的透射电镜(TEM)照片显示硫掺杂对碳纳米笼 的形貌无明显影响, 纳米笼的尺寸为 $15 \sim 30 \mathrm{~nm}$, 高分 辨透射电镜照片(HRTEM)表明笼壁石墨层约 5 10 层, 石墨化程度较好(图 1a, 1b 插图). 图 1c 的氮气吸附-脱附 等温线均为典型的 IV 型曲线, 表明样品中含有介孔, 主 要来自于纳米笼的笼内空腔及纳米笼之间的堆积孔 ${ }^{[20]}$; 图 1d 的孔分布曲线表明未掺杂碳纳米笼的孔尺寸分布 在 6 30 nm 之间, 而硫掺杂后孔分布在 8 $50 \mathrm{~nm}$ 之间, 这可能源于噻吩与苯在 $\mathrm{MgO}$ 模板上沉积行为的不同. $\mathrm{X}$ 射线光电子能谱(XPS)结果显示 $\mathrm{S}_{0} \mathrm{CNC}$ 中仅含有 $\mathrm{C}$ 和 $\mathrm{O}$
元素, 而 $\mathrm{S}_{100} \mathrm{CNC}$ 中含有 $\mathrm{C} 、 \mathrm{O}$ 和 $\mathrm{S}$ 的信号峰. 图 $1 \mathrm{f}$ 中 $\mathrm{S}_{100} \mathrm{CNC}$ 样品的 $\mathrm{S} 2 \mathrm{p}$ 谱可分为两个峰, 分别位于 163.9 和 $165.1 \mathrm{eV}$ 处, 对应于自旋耦合产生的 $2 \mathrm{p}_{3 / 2}$ 和 $2 \mathrm{p}_{1 / 2}$, 可 归属为噻吩硫 ${ }^{[14,15]}$. 由于噻吩在碳纳米笼的合成温度 $\left(800{ }^{\circ} \mathrm{C}\right)$ 下不能稳定存在, 排除了物理吸附的可能, 说 明硫主要以 $\mathrm{C}-\mathrm{S}-\mathrm{C}$ 形式掺入到碳纳米笼中.

系列样品的比表面积和元素组成见表 1 . 硫掺杂样 品的比表面积与未掺杂样品相比略有下降, 大都处于 $900 \sim 1000 \mathrm{~m}^{2} \cdot \mathrm{g}^{-1}$ 范围. XPS 分析显示硫掺杂量随前驱 物中噻吩体积比增加而升高, 可在 $0 \sim 3.45 \mathrm{at} \%$ 范围内 调变. 以上结果表明，我们成功制得了比表面积和石墨 化程度相似而硫含量可调的 SCNCs 系列样品, 这为考 察硫掺杂量对氧还原性能的影响提供了物质基础.

在 $\mathrm{O}_{2}$ 饱和的 $0.1 \mathrm{~mol} \cdot \mathrm{L}^{-1} \mathrm{KOH}$ 溶液中, 用循环伏安 法 $(\mathrm{CV})$ 、旋转圆盘电极法 $(\mathrm{RDE})$ 和旋转圆盘圆环电极法 (RRDE) 表征 $\mathrm{S}_{n} \mathrm{CNCs}$ 系列样品的电化学性能. 图 $2 \mathrm{a}$ 和 $2 \mathrm{~b}$ 分别列出了未掺杂样品 $\left(\mathrm{S}_{0} \mathrm{CNC}\right)$ 、最低硫含量样品 $\left(\mathrm{S}_{5} \mathrm{CNC}\right)$ 及最高硫含量样品 $\left(\mathrm{S}_{100} \mathrm{CNC}\right)$ 的 $\mathrm{CV}$ 和 $\mathrm{RDE}$ 曲 线. 三个纳米笼样品均有一定的催化活性, 其起始电位 比商用 $\mathrm{Pt} / \mathrm{C}$ 催化剂的 $-0.030 \mathrm{~V}$ 低 $0.115,0.107,0.122 \mathrm{~V}$. 与未掺杂的 $\mathrm{S}_{0} \mathrm{CNC}$ 相比, 低硫含量样品 $\mathrm{S}_{5} \mathrm{CNC}$ 的极限 电流更高、起始电位更靠近 $\mathrm{Pt} / \mathrm{C}$, 而高硫含量样品 $\mathrm{S}_{100} \mathrm{CNC}$ 的极限电流和起始电位比 $\mathrm{S}_{0} \mathrm{CNC}$ 略差. 图 $2 \mathrm{c}$ 和 $2 \mathrm{~d}$ 分别为 $\mathrm{S}_{5} \mathrm{CNC} 、 \mathrm{~S}_{100} \mathrm{CNC}$ 及 $\mathrm{Pt} / \mathrm{C}$ 的 RRDE 曲线及 相应的电子转移数 $(n)$ 和双氧水含量 $\left(\mathrm{HO}_{2}{ }^{-}\right)$与电位的关 系曲线. 结果显示: $\mathrm{Pt} / \mathrm{C}$ 的电子转移数 $n$ 为 3.92 , 双氧水 含量低于 $10 \%$, 这表明 $\mathrm{Pt} / \mathrm{C}$ 催化下的氧还原反应为 4 电 子过程; $\mathrm{S}_{5} \mathrm{CNC}$ 和 $\mathrm{S}_{100} \mathrm{CNC}$ 在 $-0.2 \sim-0.8 \mathrm{~V}$ 范围内的 电子转移数 $n$ 均保持在 3.2 左右, 与文献报道的硫掺杂 石墨烯的电子转移数相当 ${ }^{[13,14]}$, 双氧水含量约为 $40 \%$, 这表明在硫掺杂碳纳米笼的催化下进行 2 电子过程和 4 电子过程共存的氧还原反应过程.

图 3 为掺杂硫原子百分含量与 $\mathrm{CV}$ 起始电位和 $\mathrm{RDE}$ 峰电流密度的关系曲线. 可见，与未掺杂的 $\mathrm{S}_{0} \mathrm{CNC}$ 相 比, 硫掺杂量为约 $0.84 \mathrm{at} \%$ 样品的起始电位的正向偏移 量和峰电流密度达到最大. 随着硫含量进一步增加, 其 起始电位逐渐负向偏移, 电流密度也逐渐下降. 当硫掺 杂量在 $1.61 \sim 3.45 \mathrm{at} \%$ 时, 样品的起始电位甚至略低于 $\mathrm{S}_{0} \mathrm{CNC}$ 而其峰电流密度相当. 这个结果表明硫掺杂碳纳 米笼的氧还原催化性能与硫含量密切相关, 低硫含量促 进氧还原性能，而高硫含量劣化氧还原性能.

一般而言，掺杂和碳材料自身的拓扑缺陷均可调变 $\mathrm{sp}^{2}$ 碳的电子结构, 从而使其具有一定的氧还原活性 ${ }^{[22]}$. $\mathrm{S}_{0} \mathrm{CNC}$ 具有较石墨烯更多的缺陷，具有较好的氧还原催 化性能，在起始电位上优于文献报道的石墨烯(通常约 为 $-0.15 \sim-0.20 \mathrm{~V}$ vs. $\mathrm{Ag} / \mathrm{AgCl})^{[14]}$. 我们的前期研究 结果表明，掺杂碳基纳米材料的氧还原活性源于 $\pi$ 电子 的活化、产生利于 $\mathrm{O}_{2}$ 分子吸附的带正电的活性位 ${ }^{[12,19]}$. 

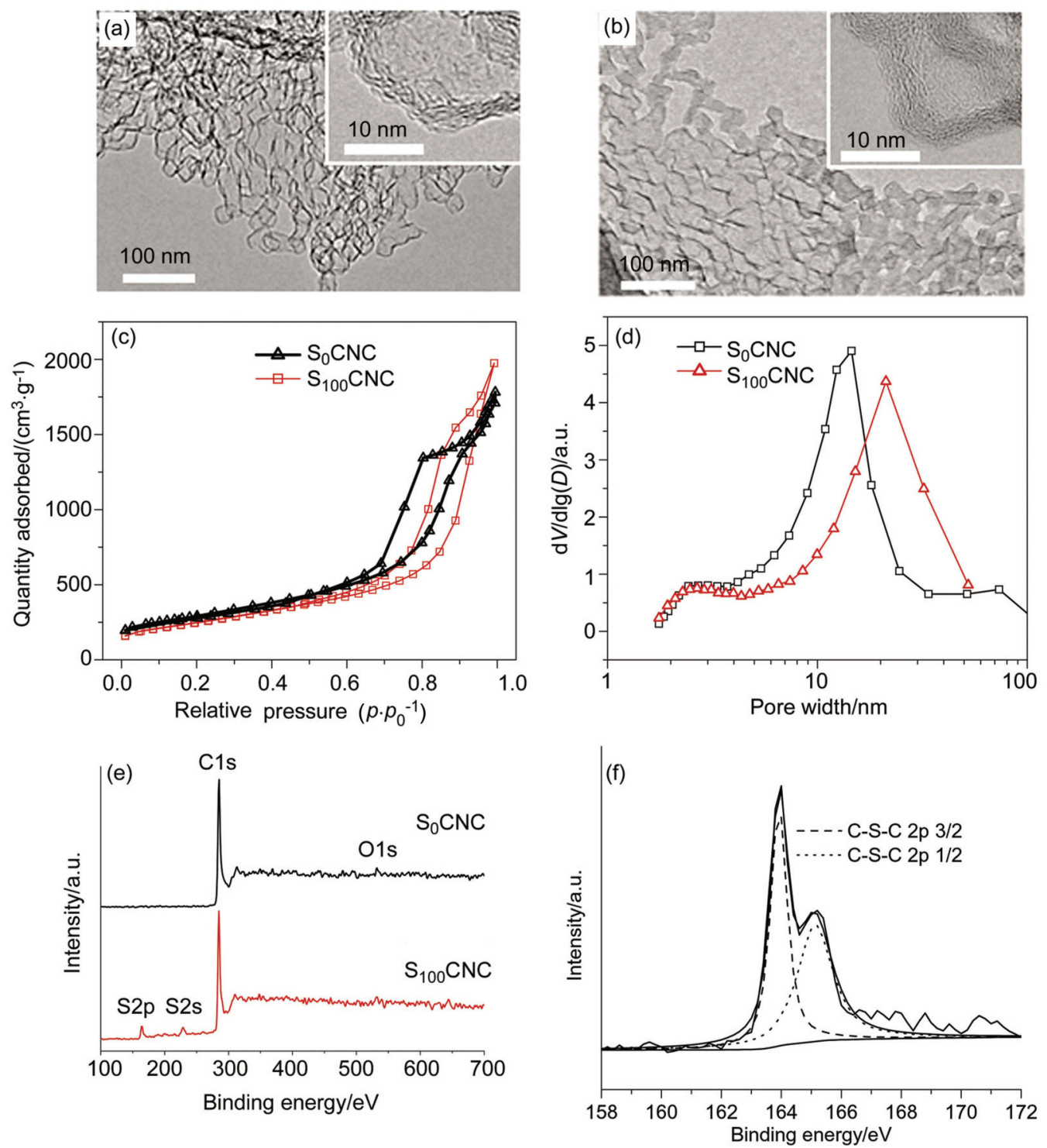

图 $1 \mathrm{~S}_{n} \mathrm{CNCs}$ 的结构和成分表征. (a, b) $\mathrm{S}_{0} \mathrm{CNC}$ 和 $\mathrm{S}_{100} \mathrm{CNC}$ 的(HR)TEM 照片; (c,d) $\mathrm{S}_{0} \mathrm{CNC}$ 和 $\mathrm{S}_{100} \mathrm{CNC}$ 的吸附-脱附等温线以及由 $\mathrm{BJH}$ 方法计算吸 附支曲线得到的孔分布图; (e) $\mathrm{S}_{0} \mathrm{CNC}$ 和 $\mathrm{S}_{100} \mathrm{CNC}$ 的 XPS 全谱; (f) $\mathrm{S}_{100} \mathrm{CNC}$ 的 $\mathrm{S} 2 \mathrm{p}$ 谱

Figure 1 The structure and component characterization of $\mathrm{S}_{n} \mathrm{CNCs}$. $(\mathrm{a}, \mathrm{b})$ Typical (HR)TEM images of $\mathrm{S}_{0} \mathrm{CNC}$ and $\mathrm{S}_{100} \mathrm{CNC}$. (c, d) Nitrogen adsorption/desorption isotherms of $\mathrm{S}_{0} \mathrm{CNC}$ and $\mathrm{S}_{100} \mathrm{CNC}$ and corresponding pore-size distribution plots calculated by the $\mathrm{BJH}$ method from the adsorption branch isotherms. (e) XPS survey spectra of $\mathrm{S}_{0} \mathrm{CNC}$ and $\mathrm{S}_{100} \mathrm{CNC}$. (f) $\mathrm{S} 2 \mathrm{p}$ deconvoluted spectrum of $\mathrm{S}_{100} \mathrm{CNC}$

表 $1 \mathrm{~S}_{n} \mathrm{CNCs}$ 的相对原子含量及比表面积

Table 1 Surface atomic concentrations (from XPS) and BET surface areas of the series of $\mathrm{S}_{n} \mathrm{CNC}$ samples

\begin{tabular}{ccccc}
\hline Sample & $\mathrm{C} / \mathrm{at} \%$ & $\mathrm{~S} / \mathrm{at} \%$ & $\mathrm{O} / \mathrm{at} \%$ & $\mathrm{BET} /\left(\mathrm{m}^{2} \bullet \mathrm{g}^{-1}\right)$ \\
\hline $\mathrm{S}_{0} \mathrm{CNC}$ & 97.65 & - & 2.35 & 1034 \\
$\mathrm{~S}_{5} \mathrm{CNC}$ & 97.64 & 0.84 & 1.52 & 978 \\
$\mathrm{~S}_{10} \mathrm{CNC}$ & 96.81 & 1.12 & 2.07 & 870 \\
$\mathrm{~S}_{15} \mathrm{CNC}$ & 97.06 & 1.61 & 1.33 & 978 \\
$\mathrm{~S}_{25} \mathrm{CNC}$ & 94.64 & 2.53 & 2.83 & 934 \\
$\mathrm{~S}_{50} \mathrm{CNC}$ & 94.95 & 3.08 & 1.97 & 902 \\
$\mathrm{~S}_{75} \mathrm{CNC}$ & 94.48 & 3.40 & 2.12 & 967 \\
$\mathrm{~S}_{100} \mathrm{CNC}$ & 93.88 & 3.45 & 2.68 & 902 \\
\hline
\end{tabular}

硫原子具有 6 个外层电子，其电负性(2.58)略高于碳 (2.55). 因此, 少量硫掺杂有利于增加碳的电子密度, 其 稍高于碳的电负性使相邻碳原子带少量正电荷，从而促 进氧还原活性的改善. 由于 $\mathrm{S}$ 和 $\mathrm{C}$ 之间的电负性相差很 小, $\mathrm{S}$ 掺杂对氧还原性能的提高有限. 此外, $\mathrm{S}$ 掺杂量较 大时引入的拓扑缺陷会使导电性下降, 不利于氧还原过 程, 这可能是氧还原性能随掺杂 $\mathrm{S}$ 含量增加先有所改善 再逐渐变差的原因.

众所周知，燃料电池的阴极催化剂必须具有良好的 抗甲醇渗透性能和稳定性. 图 $4 \mathrm{a}$ 为 $\mathrm{S}_{100} \mathrm{CNC}$ 和 $\mathrm{Pt} / \mathrm{C}$ 的 抗甲醇渗透能力比较, 当甲醇引入 $\mathrm{KOH}$ 溶液后, $\mathrm{S}_{100} \mathrm{CNC}$ 受影响很小, 而 $\mathrm{Pt} / \mathrm{C}$ 的氧还原电流下降达 $50 \%{ }^{[23]}$, 这表明 $\mathrm{SCNC}$ 电催化剂具有优良的抗甲醇渗透 

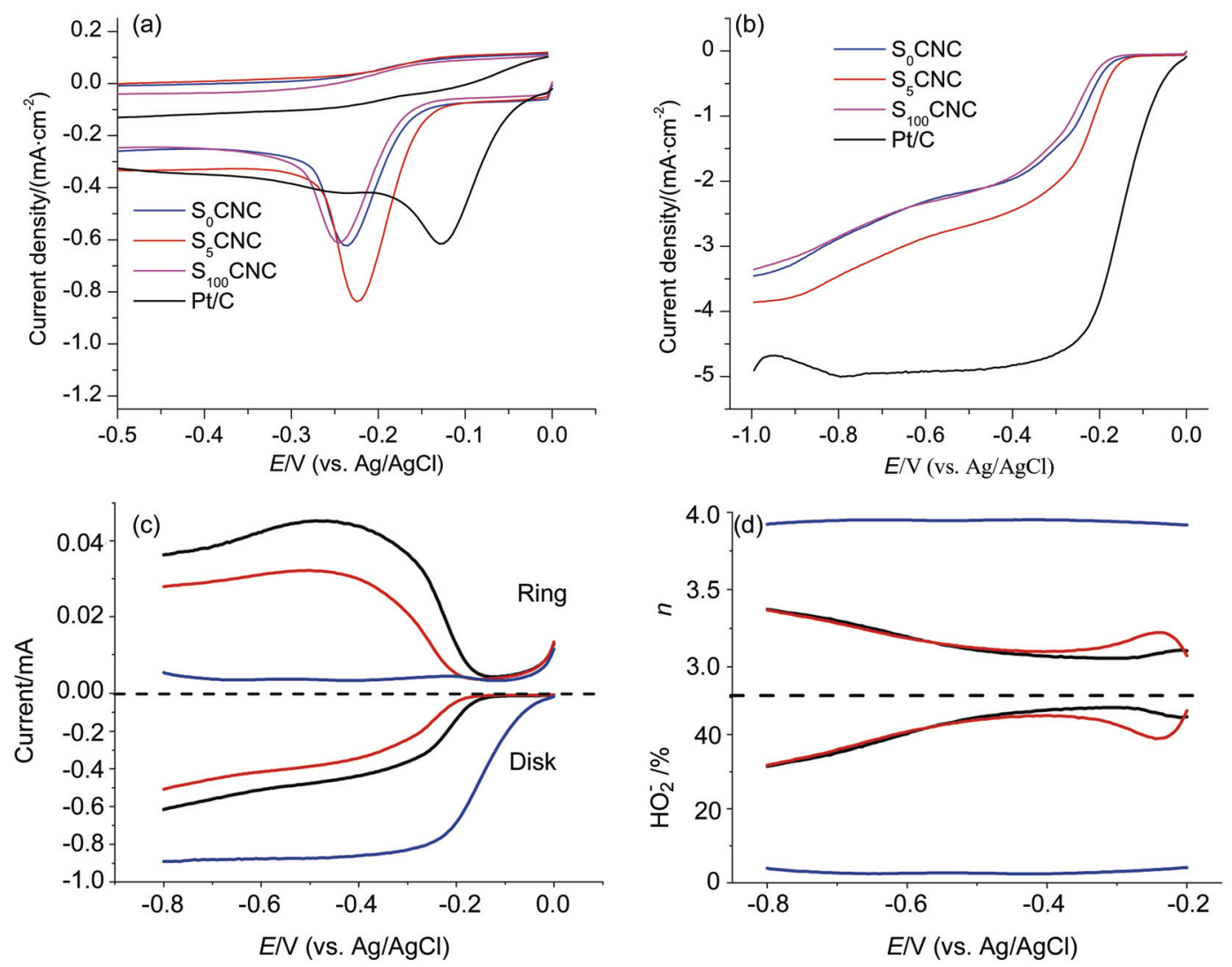

图 2 (a, b) $\mathrm{S}_{0} \mathrm{CNC}, \mathrm{S}_{5} \mathrm{CNC}, \mathrm{S}_{100} \mathrm{CNC}$ 在 $\mathrm{O}_{2}$ 饱和的 $0.1 \mathrm{~mol} \cdot \mathrm{L}^{-1} \mathrm{KOH}$ 电解液中的 $\mathrm{CV}$ 和 $\mathrm{RDE}$ 曲线. 扫速为 $10 \mathrm{mV} \cdot \mathrm{s}^{-1} ;(\mathrm{c}, \mathrm{d}) \mathrm{S}_{5} \mathrm{CNC}($ 黑色)、 $\mathrm{S}_{100} \mathrm{CNC}$ (红色)的 RRDE 曲线及相应的电子转移数和双氧水含量. (a d )中也提供了商用 $\mathrm{Pt} / \mathrm{C}$ 催化剂 $(20 \mathrm{wt} \% \mathrm{Pt}$ )的相应表征结果(蓝色)作对比.

Figure 2 (a, b) $\mathrm{CV}$ and $\mathrm{RDE}$ curves of $\mathrm{S}_{0} \mathrm{CNC}, \mathrm{S}_{5} \mathrm{CNC}, \mathrm{S}_{100} \mathrm{CNC}$ in $\mathrm{O}_{2}$-saturated $0.1 \mathrm{~mol} \cdot \mathrm{L}^{-1} \mathrm{KOH}$ at scan rates of $10 \mathrm{mV} \cdot \mathrm{s}^{-1}$. (c, d) $\mathrm{RRDE}$ curves of $\mathrm{S}_{5} \mathrm{CNC}$ (black), $\mathrm{S}_{100} \mathrm{CNC}$ (red) and the corresponding transferred electron number (n)/percentage of peroxide. For comparison, the characterizations on commercial $\mathrm{Pt} / \mathrm{C}$ catalyst $(20 \mathrm{wt} \% \mathrm{Pt})$ are also provided in $(\mathrm{a} \sim \mathrm{d})(\mathrm{blue})$

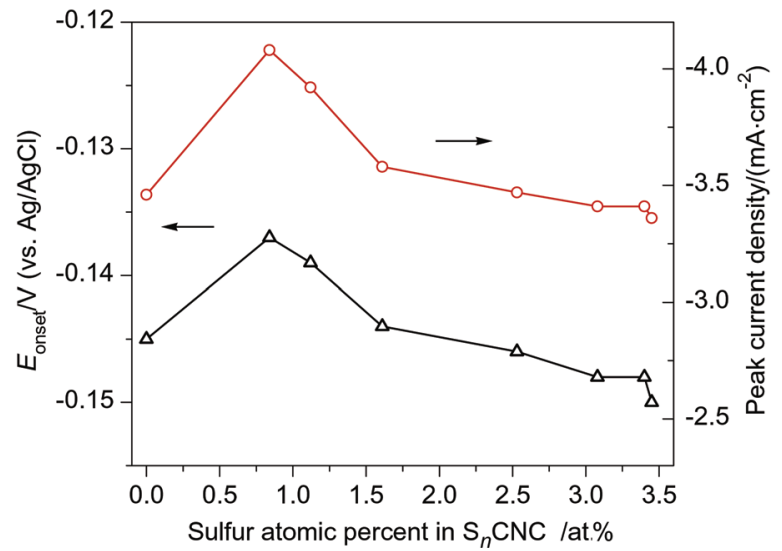

图 $3 \mathrm{~S}_{n} \mathrm{CNC}$ 系列样品的硫原子百分含量与 $\mathrm{CV}$ 起始电位(黑)和 $\mathrm{RDE}$ 峰电流密度(红)的关系曲线

Figure 3 The correlation of S atomic percent of $\mathrm{S}_{n} \mathrm{CNC}$ samples vs. $\mathrm{CV}$ onset potentials (black) and RDE peak current densities (red)

的能力. 图 $4 \mathrm{~b}$ 为 $\mathrm{S}_{100} \mathrm{CNC}$ 和 $\mathrm{Pt} / \mathrm{C}$ 的稳定性比较, 经过 $24 \mathrm{~h}$ 测试后 $\mathrm{S}_{100} \mathrm{CNC}$ 仍保留着 $95 \%$ 的电流密度, 而 $\mathrm{Pt} / \mathrm{C}$ 仅保留了 $80 \%$ 的电流密度, 即 $\mathrm{SCNC}$ 具有良好的稳定 性.

\section{3 结论}

以 $\mathrm{MgO}$ 为模板, 以噻吩和苯为前驱物, 通过 $\mathrm{CVD}$ 法合成了比表面积大、硫掺杂量在 $0 \sim 3.45$ at $\%$ 范围内可 调、石墨化程度良好的硫掺杂碳纳米笼, 在碱性环境中 表现出良好的氧还原活性、优良的抗甲醇渗透性能和稳 定性. 系统考察了不同硫掺杂量对氧还原催化性能的影 响, 硫含量为 $0.84 \mathrm{at} \%$ 的 SCNCs 具有最佳的氧还原性 能, 随着硫掺杂量增多其催化性能逐渐变差, 当硫的掺 杂量高于 $1.61 \mathrm{at} \%$ 时甚至劣于纯碳纳米笼. 这对于通过 调变掺杂元素种类、含量及掺杂构型设计和构建碳基无 金属氧还原催化剂提供了有益参考.

\section{4 实验部分}

\section{1 材料制备}

通过原位 $\mathrm{MgO}$ 模板法, 以噻吩和苯为前驱物在 800 ${ }^{\circ} \mathrm{C}$ 下合成硫掺杂碳纳米笼( $\left.\mathrm{SCNCs}\right)$. 取 $1.5 \mathrm{~g}$ 碱式碳酸镁 粉末平推于管式炉中心, 在 $\mathrm{Ar}$ 气氛下以 $10{ }^{\circ} \mathrm{C} / \mathrm{min}$ 升温 

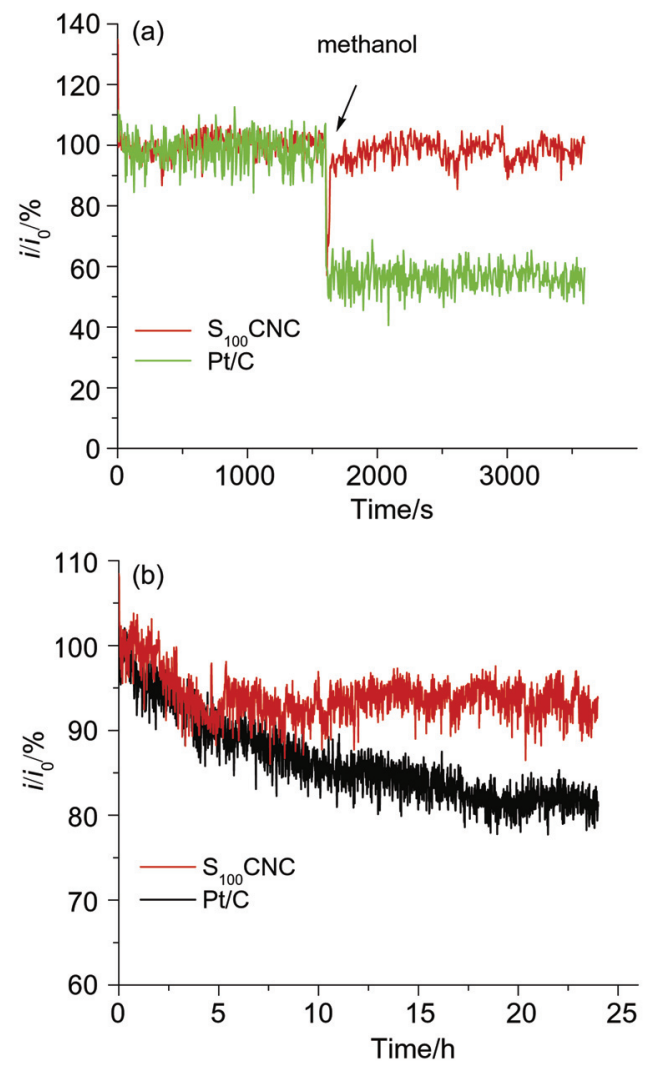

图 4 (a) $\mathrm{S}_{100} \mathrm{CNC}$ 和 $\mathrm{Pt} / \mathrm{C}$ 的抗甲醇渗透性能; (b)电流-时间曲线. $i_{0}$ 为 初始电流, 偏置电位为 $-0.4 \mathrm{~V}, 0.1 \mathrm{~mol} \cdot \mathrm{L}^{-1} \mathrm{KOH}$.

Figure 4 Comparison of the methanol crossovers (a) and ORR stabilities (b) for $\mathrm{S}_{100} \mathrm{CNC}$ and $\mathrm{Pt} / \mathrm{C}$ at $-0.4 \mathrm{~V}$ bias voltage and $0.1 \mathrm{~mol} \cdot \mathrm{L}^{-1}$ $\mathrm{KOH}$ solution. $i_{0}$ defines the initial current

到 $800{ }^{\circ} \mathrm{C}$. 用注射洜在 $30 \mathrm{~min}$ 内向反应系统中注入 1 $\mathrm{mL}$ 配制好的前驱物, 然后在 $\mathrm{Ar}$ 气氛下自然冷却至室 温. 将产物在 $6 \mathrm{~mol} / \mathrm{L}$ 的盐酸中搅拌 $24 \mathrm{~h}$ 除去 $\mathrm{MgO}$ 模板, 过滤并用蒸馏水和乙醇多次洗涤后在 $60{ }^{\circ} \mathrm{C}$ 下干燥 $12 \mathrm{~h}$, 即得 SCNCs 样品.

\section{2 材料表征}

$\mathrm{SCNCs}$ 的形貌和结构通过高分辨率透射电镜 (HRTEM, JEM-2100, JEOL, 操作电压 $200 \mathrm{kV}$ ) 观察, 比 表面积和孔分布用氮气吸脱附测试仪[Micromeritics ASAP 2010, 液氮(77 K)]测定, 孔分布由 Barrett-JoynerHalenda(BJH) 法计算吸附支曲线得到，比表面积由 Brunauer-Emmett-Teller(BET)方法得到. 元素的化学态 及含量信息通过 $\mathrm{X}$ 射线光电子能谱 (XPS, VG ESCALAB MKII)进行表征.

\section{3 电化学性能表征}

将 $2 \mathrm{mg}$ 催化剂加入 $1 \mathrm{~mL}$ 乙醇水溶液(体积比为 1 : 4), 超声 $1 \mathrm{~h}$ 使催化剂分散均匀. 取 $10 \mu \mathrm{L}$ 上述悬浮液滴 涂到玻碳电极 $(\mathrm{GC}$, 直径 $5 \mathrm{~mm}$ )表面, 再滴加 $2 \mu \mathrm{L}$ Nafion (Dupont, 5\%), 室温晾干. 所有的电化学性能测
试均在 $\mathrm{CHI} 760 \mathrm{C}$ 电化学工作站上完成, 以 $\mathrm{Ag} / \mathrm{AgCl}$ 为 参比电极, Pt 丝电极为对电极, 涂有催化剂的玻碳电极 为工作电极组成三电极系统, 电解液为 $0.1 \mathrm{~mol} \cdot \mathrm{L}^{-1}$ $\mathrm{KOH} . \mathrm{CV}, \mathrm{RDE}$ 及 RRDE 曲线均在预先通入 $20 \min \mathrm{O}_{2}$ 的 $0.1 \mathrm{~mol} \cdot \mathrm{L}^{-1} \mathrm{KOH}$ 中测定，扫描速率为 $10 \mathrm{mV} \cdot \mathrm{s}^{-1}$, 扫 描范围为 $0 \sim-1 \mathrm{~V}$. RDE 及 RRDE 转速为 $2500 \mathrm{r} / \mathrm{min}$, RRDE 的环电压设置为 $0.5 \mathrm{~V}$. 电子转移数及双氧水产 率分别由公式 $n=4 I_{\text {disk }} /\left(I_{\text {disk }}+I_{\text {ring }} / N\right)$ 和 $\mathrm{HO}_{2}{ }^{-}=$ $200 I_{\text {ring }} / N /\left(I_{\text {disk }}+I_{\text {ring }} / N\right)^{[24]}$ 得到, 收集系数 $N=0.26$. 稳态 计时电流法用于测量催化剂抗甲醇渗透性能及稳定性, 偏置电位为 $-0.4 \mathrm{~V}$. 测试过程中不断向电解液中通入 氧气并摚拌. 抗甲醇渗透实验在测试进行到 $1600 \mathrm{~s}$ 时加 入 $2 \%$ 体积比的甲醇.

\section{References}

[1] Chu, S.; Majumdar, A. Nature 2012, 488, 294

[2] Zhang, D.; Zhang, C.-Z.; Mu, D.-B.; Wu, B.-R.; Wu, F. Acta Chim. Sinica 2013, 71, 1101. (张栋, 张存中, 穆道斌, 吴伯荣, 吴锋, 化 学学报, 2013, 71, 1101.)

[3] Yang, C. J. Energy Policy 2009, 37, 1805.

[4] Lu, Z.-J.; Xu, M.-W.; Bao, S.-J.; Chai, H. Acta Chim. Sinica 2013, 71, 957. (鲁振江, 徐茂文, 包淑娟, 柴卉, 化学学报, 2013, 71, 957)

[5] Gasteiger, H. A.; Markovic, N. M. Sicence 2009, 324, 48.

[6] Zheng, L.-Z.; Tao, K.; Xiong, L.-Y.; Ye, D.; Han, K.; Ji, Y. Acta Chim. Sinica 2012, 70, 2342. (郑龙珍, 陶埕, 熊乐艳, 叶丹, 韩奎, 纪忆, 化学学报, 2012, 70, 2342.)

[7] Yang, L.-J.; Zhao, Y.; Chen, S.; Wu, Q.; Wang, X.-Z.; Hu, Z. Chin. J. Catal. 2013, 34, 1986. (杨立军, 赵宇, 陈盛, 吴强, 王喜章, 胡 征, 催化学报, 2013, 34, 1986.)

[8] Gong, K.-P.; Du, F.; Xia, Z.-H.; Durstock, M.; Dai, L.-M. Science 2009, 323, 760 .

[9] Qu, L.-T.; Liu, Y.; Baek, J. B.; Dai, L.-M. ACS Nano 2010, 4, 1321.

[10] Liu, R.-L.; Wu, D.-P.; Feng, X.-L.; Müllen, K. Angew. Chem. Int. Ed. 2010, 49, 1

[11] Lyth, S. M.; Nabae, Y.; Moriya, S.; Kuroki, S.; Kakimoto, M.; Ozaki, J.; Miyata, S. J. Phys. Chem. C 2009, 113, 20148.

[12] Yang, L.-J.; Jiang, S.-J.; Zhao, Y.; Zhu, L.; Chen, S.; Wang, X.-Z.; Wu, Q.; Ma, J.; Ma, Y.-W.; Hu, Z. Angew. Chem. Int. Ed. 2011, 50, 7132 .

[13] Yang, Z.; Yao, Z.; Li, G.-F.; Fang, G.-Y.; Nie, H.-G.; Liu, Z.; Zhou, X.-M.; Chen, X.; Huang, S.-M. ACS Nano 2012, 6, 205.

[14] Liang, J.; Jiao, Y.; Jaroniec, M.; Qiao, S.-Z. Angew. Chem. Int. Ed. 2012, 51, 11496.

[15] Yang, S.-B.; Zhi, L.-J.; Tang, K.; Feng, X.-L.; Maier, J.; Müllen, K. Adv. Funct. Mater. 2012, 22, 3634.

[16] Yang, D.-S.; Bhattacharjya, D.; Inamdar, S.; Park, J.; Yu, J.-S. J. Am. Chem. Soc. 2012, 134, 16127.

[17] Yao, Z.; Nie, H.-G.; Yang, Z.; Zhou, X.-M.; Liu, Z.; Huang, S.-M. Chem. Commun. 2012, 48, 1027.

[18] Chen, Y.; Yang, X.-C.; Liu, Y.-J.; Zhao, J.-X.; Cai, Q.-H.; Wang, X.-Z. J. Mol. Graph. Model. 2013, 39, 126.

[19] Zhao, Y.; Yang, L.-J.; Chen, S.; Wang, X.-Z.; Ma, Y.-W.; Wu, Q.; Jiang, Y.-F.; Qian, W.-J.; Hu, Z. J. Am. Chem. Soc. 2013, 135, 1201.

[20] Xie, K.; Qin, X.-T.; Wang, X.-Z.; Wang, Y.-N.; Tao, H.-S.; Wu, Q.; Yang, L.-J.; Hu, Z. Adv. Mater. 2012, 24, 347.

[21] Chen, S.; Bi, J.-Y.; Zhao, Y.; Yang, L.-J.; Zhang, C.; Ma, Y.-W.; Wu, Q.; Wang, X.-Z.; Hu, Z. Adv. Mater. 2012, 24, 5593.

[22] Zhang, L.-P.; Niu, J.-B.; Dai, L.-M.; Xia, Z.-H. Langmuir 2012, 28, 7542 .

[23] Liu, F.-Q.; Wang, C.-Y. J. Electrochem. Soc. 2007, 154, B514.

[24] Liang, Y.-Y.; Wang, H.-L.; Zhou, J.-G.; Li, Y.-G.; Wang, J.; Regier, T.; Dai, H.-J. J. Am. Chem. Soc. 2012, 134, 3517. 